\title{
El turismo en la región de Los Lagos: experiencias del Pueblo Mapuce y reflexiones en el contexto de la pandemia
}

\author{
María Daniela Rodríguez \\ Universidad Nacional de Comahue \\ Consejo Nacional de Investigaciones Científicas y Técnicas \\ @ [ dany_bolivar@hotmail.com ]
}

Anabela Ivana Cadiz

Universidad Nacional de Comahue Consejo Nacional de Investigaciones Científicas y Técnicas

@ [ cadizanabela2084@gmail.com ]

Cita sugerida: Rodríguez, M. D. y Cadiz, A. I. (2021). El turismo en la región de Los Lagos: experiencias del Pueblo Mapuce y reflexiones en el contexto de la pandemia. Revista Huellas, Volumen 25, № 2, Instituto de Geografía, EdUNLPam: Santa Rosa. Recuperado a partir de: http://cerac.unlpam.edu.ar/ index.php/huellas

DOI: http://dx.doi.org/10.19137/huellas-2021-2519

\begin{abstract}
Resumen
El artículo hace un recorrido sobre el desarrollo del turismo en la región de Los Lagos (Provincia de Neuquén) e indaga sobre las experiencias del pueblo Mapuce en el norte, centro y sur de la región mencionada. En un contexto marcado por la pandemia COVID-19, el interrogante que motoriza el trabajo es ¿En qué lugar de prioridad se colocó a las comunidades mapuce que sostienen servicios turísticos en estos territorios? Se propone identificar la situación socioeconómica en los territorios turísticos de la región de Los Lagos y en las comunidades mapuce que desarrollan esta actividad, en un contexto marcado por la pandemia COVID-19. El trabajo parte de reconocer la historia de exclusión, marginalidad y desigualdad social del pueblo mapuce a partir de las políticas públicas, la configuración territorial y las actividades productivas impulsadas por el Estado y su rol en el desarrollo reciente de iniciativas autónomas vinculadas al turismo. Se emplea una metodología de tipo cualitativa a partir de información secundaria y trabajo de campo en el territorio.
\end{abstract}

Palabras clave: región de Los Lagos; turismo; pueblo Mapuce; desigualdad; pandemia 


\title{
Tourism in the Los Lagos Region: experiences of the Mapuce people and reflections in the context of the pandemic
}

\begin{abstract}
This article deals with the development of tourism in Los Lagos region (Neuquén Province) and inquiries about the experiences of the Mapuce people in the north, center and south of the aforementioned region. In the context of the COVID-19 pandemic, the question that guides the work is: In which order of priority were Mapuce communities that provide tourist services in these territories placed? This work aims at identifying the socioeconomic condition in the tourist territories of Los Lagos Region and in the Mapuce communities that carry out this activity in the context of the COVID-19 pandemic. The work starts from recognizing the history of exclusion, marginality and social inequality of the Mapuce people resulting from public policies, territorial configuration and productive activities promoted by the State and its role in the recent development of autonomous initiatives related to tourism. A qualitative methodology is used on the basis of secondary information and field work in the territory.
\end{abstract}

Key words: Los Lagos region; tourism; Mapuce people; inequality; pandemic

\section{0 turismo na região de Los Lagos: experiências do Povo Mapuce e reflexões no contexto da pandemia}

\begin{abstract}
Resumo
Este artigo percorre o desenvolvimento turístico na região de Los Lagos (província de Neuquén) e indaga sobre as experiências do povo Mapuce no norte, centro e sul da região citada. Em um contexto de pandemia COVID-19, o interrogante que motiva este trabalho é: que prioridade se deu às comunidades mapuce que sustentam serviços turísticos nestes territórios? Propõem-se identificar a situação social e econômica nos territórios turísticos da região de Los Lagos e nas comunidades mapuce que desenvolvem esta atividade em um contexto de pandemia COVID-19. O trabalho parte de reconhecer a história de exclusão, marginalidade e desigualdade social do povo Mapuce a partir das políticas públicas, a configuração territorial e as atividades produtivas impulsadas pelo Estado e seu papel no desenvolvimento recente de iniciativas autônomas vinculadas ao turismo. Emprega-se uma metodologia de tipo qualitativa a partir da informação secundaria e o trabalho de campo no território.
\end{abstract}

Palavras-chave: Região de Los Lagos; turismo; povo Mapuce; desigualdade; pandemia 


\section{Introducción}

A lo largo de la historia, distintas enfermedades han azotado a la humanidad: viruela, sarampión, gripe española, peste negra y VIH. Según la Organización Mundial de la Salud (OMS), para que una enfermedad se denomine pandemia, debe extenderse de manera acelerada por todo el mundo y afectar a un gran número de personas que no es inmune al desarrollo del mismo. Desde finales de 2019 e inicios de 2020, vivimos tiempos únicos en la historia de la humanidad ante la llegada de la Pandemia COVID-19. La coyuntura actual vino a modificar la "normalidad" en la vida de las personas, recluyéndolas a los hogares y a respetar determinadas conductas de aislamiento y distanciamiento social para salvaguardar sus vidas y de esa forma evitar el colapso del sistema sanitario. Lo que hasta ayer era "normal", nos referimos a la movilidad de la población por placer, ocio, dispersión, consumo, recreación, motivaciones necesarias para el funcionamiento de la actividad turística, se vieron restringidas y por lo tanto el turismo afectado en gran parte del año 2020. Esta situación cambió de forma paulatina hacia fin de año. En este sentido, los sitios turísticos se han preparado para recibir visitantes durante la temporada estival, a partir del diseño de estrictos protocolos sanitarios y trabajo mancomunado entre municipio, provincia, nación y el sector en cuestión.

En la provincia de Neuquén, Patagonia Argentina, el denominado corredor de Los Lagos ${ }^{2}$ o Ruta de los Siete Lagos, se considera un área turística de gran relevancia a nivel nacional y mundial. Más allá de la identidad turística propia del corredor antes mencionado, aquí se trabaja con la concepción de región de Los Lagos, para hacer referencia al área geográfica que se extiende por el borde occidental de la Cordillera de los Andes, dentro de los límites jurisdiccionales del Departamento Los Lagos, Lácar, Huiliches y Aluminé, provincia de Neuquén y en su interior se encuentran los ejidos municipales de Villa La Angostura, San Martín de los Andes, Junín de los Andes y Aluminé. Una región en la cual destaca el turismo como actividad organizadora del territorio, estructuradora de la sociedad y dinamizadora de la economía regional. En la investigación, se considera la región como una construcción social, cuya extensión está determinada por la complejidad socio-económica, territorial y cultural, así como por los actores sociales, los intereses, las relaciones intra y extrarregionales y las lógicas de valorización de los recursos naturales.

Tal como se mencionó, el turismo ha sido una de las principales actividades económicas, en cuanto a los atractivos turísticos que presenta, los requerimientos de la actividad, generación de empleo, ingresos económicos y flujos de turistas nacionales e internacionales. Es decir, una estructura organizativa y territorial que fue diseñada desde mediados del 
siglo XX, para satisfacer las necesidades de ocio, disfrute, recreación del visitante y que contribuyó a (re) orientar el perfil productivo de la región. Se puede decir que, el turismo, generó la refuncionalización de los territorios y la valorización diferencial de la naturaleza-cultura, que bajo una lógica predominantemente utilitarista y economicista se puso al servicio del mercado (March, 2013). En territorios marcados por la desigualdad estructural, el primer interrogante es iqué impacto tuvo la pandemia en el desarrollo turístico de la región de Los Lagos? Se infiere que el impacto fue diferencial en cada territorio, si se considera el espacio rural-urbano, los ejidos municipales, la jurisdicción del Parque Nacional Lanín o las comunidades mapuce que viven en esta región. A grandes rasgos, se reconoce la importancia de San Martín de los Andes y Villa La Angostura en la dinámica turística de la región mencionada. Asimismo, es necesario remarcar que se trata de los centros turísticos más importantes de la provincia del Neuquén, que desarrollan actividades durante todo el año y el mercado turístico que allí se genera, repercute en toda la región de Los Lagos. Es por ello que, es fundamental revisar lo que sucede en estas localidades como epicentro del turismo y generadora de efectos en toda la región y luego centrar la atención en las experiencias turísticas de las comunidades mapuce del sur, centro y norte de la región mencionada.

Sin lugar a dudas, la retracción económica por el contexto COVID-19, llevó a la emergencia del sector turístico en la provincia de Neuquén y a la creación de un fondo de inversión destinado a acompañar al sector afectado. A pesar de que el gobierno provincial demostró su capacidad para acompañar las medidas sanitarias y económicas adoptadas a nivel nacional, el turismo no fue un tema prioritario en la agenda política del Estado. Sin embargo, la fuerte presión ejercida por los prestadores turísticos ante la prolongada inactividad, llevó al gobierno neuquino a diseñar el Plan de Reactivación Turística 2020-2021 (resolución provincial 56/20, agosto del año 2020), autorizando el desplazamiento con fines turísticos entre localidades libres de COVID-19 que estuvieran dentro de una misma microrregión turística (Contino, 2020). Si bien, esto permitió una cierta movilidad de la población entre microrregiones, no fue significativo desde el punto de vista económico, lo cual es totalmente lógico para una región que ha dependido del flujo de turismo nacional e internacional. Hacia diciembre, las medidas se flexibilizaron para el sector turístico y se habilitó la recepción de visitantes de cualquier punto del país, con el cumplimiento de los protocolos sanitarios establecidos. De todas formas, habrá que esperar la finalización de la temporada estival del año 2021 para realizar una evaluación de lo acontecido.

Este panorama incierto lleva a preguntarnos ise contempló la especificidad del sector turístico en la región de Los Lagos? ¿En qué lugar de 
prioridad se colocó a las comunidades mapuce que sostienen servicios turísticos en estos territorios? o acaso esta situación ¿puso nuevamente en evidencia que los grupos más postergados continúan siendo excluidos o no contemplados por el Estado provincial? He aquí un punto central del artículo. En la provincia de Neuquén se registran oficialmente más de cincuenta comunidades mapuce, de las cuales un número importante se encuentran asentadas en la zona de cordillera y precordillera andina, sitio en el cual existe un gran desarrollo turístico y del cual estas participan minoritariamente. En tal sentido, existen experiencias turísticas desarrolladas por este pueblo, que han demostrado que es posible llevar a cabo turismo en territorio mapuce, revalorizando el sentido del lugar, portador de significados e identidades (Albet i Mas, 2001), la cultura, los saberes ancestrales, las prácticas comunitarias y la memoria colectiva. Poniendo el foco de atención en el resguardo del territorio, el cuidado del bosque, el agua y la biodiversidad. Este artículo busca realizar una revisión de la construcción del territorio turístico en la región de Los Lagos, desde una mirada diacrónica desde mediados del siglo pasado e indagar particularmente en algunas iniciativas y experiencias turísticas desarrolladas por este colectivo. Por otro lado, desde una mirada sincrónica, se propone identificar la situación socioeconómica en los territorios turísticos de la región de Los Lagos y en las comunidades mapuce que desarrollan esta actividad en el contexto de la pandemia COVID-19.

\section{Consideraciones metodológicas}

La investigación consiste en un diseño del tipo descriptivo exploratorio, a partir del cual se presentan resultados preliminares. La metodología de análisis que se propone es de tipo cualitativa, asentada en la revisión de bibliografía específica e información secundaria suministrada por periódicos de alcance regional y nacional durante los años 2020 y 2021 . También se utilizan fuentes de información primaria relevada durante el trabajo de campo realizado entre los años 2012 e inicios del 2020. La búsqueda, selección y jerarquización de la información se conjuga con el trabajo de campo, realizado en torno a las experiencias turísticas de las comunidades mapuce en la región de Los Lagos, así como proyectos y programas asociados al desarrollo del turismo en el área mencionada. Esta instancia de la investigación, se nutrió de observaciones de la dinámica turística en la región de Los Lagos, entrevistas a referentes de las comunidades mapuce, participación de talleres y celebraciones en comunidades mapuce, entrevistas estructuradas, semi-estructuradas y encuentros virtuales en el año 2020 con referentes de comunidades mapuce e integrantes del Parque Nacional Lanín en el marco del proyecto que participa una de las autoras ${ }^{3}$. 
La mirada interdisciplinaria ${ }^{4}$ y el diálogo de saberes fueron fundamentales para entender la dinámica de los territorios turísticos cordilleranos, en los cuales es evidente la disputa en torno a la valorización de los recursos naturales y la apropiación de los territorios que genera la actividad turística.

\section{El turismo en la región de Los Lagos ¿configurador territorial de la desigualdad?}

Desde la mirada de la Geografía e Historia Regional, la región es entendida como una categoría de análisis, no circunscripta a límites fijos y estáticos, sino como un espacio singular y único, cuya extensión estará determinada y/o condicionada por la dinámica y la complejidad del propio tema de estudio (Bandieri, 2005; De Jong, 2009). En este sentido, la región excede los límites jurídicos-administrativos y debe pensarse como un proceso de construcción social, en el cual los elementos que la componen, es decir, la historia, rasgos socioculturales, políticos, económicos y ambientales, así como los actores sociales, se van transformado a lo largo del tiempo y con ello el territorio que habitan. Tal como señala Valenzuela (2012, p.9) "la región no es solo una construcción social: es un proceso históricamente contingente y permanentemente abierto, inacabado y en transformación", con diversas escalas espacio-temporales.

La región de Los Lagos, se encuentra localizada geográficamente dentro de los límites jurisdiccionales del Departamento Los Lagos, Lácar, Huiliches y Aluminé, provincia de

Neuquén y en su interior se encuentran los ejidos municipales de Villa La Angostura, San Martín de los Andes, Junín de los Andes y Aluminé (Figura $\mathrm{N}^{\circ} 1$ ).

Destacada por la belleza paisajística que le imprimen las condiciones naturales de la ecorregión del bosque andino patagónico y las condiciones socio-culturales e identidad del lugar, aquí las actividades agrícolas-ganaderas, el turismo y la lógica gubernamental de conservación de la naturaleza y protección del ambiente, se convirtieron en los ejes fundamentales en la re-estructuración y ordenamiento del territorio, así como de las formas de vida, la organización socio-cultural y económica de la población.

La potencialidad estética, paisajística y simbólica de la región de Los Lagos, la posicionó históricamente como un territorio estratégico para el Estado nacional, hecho que se tradujo en la elaboración y ejecución de políticas públicas tendientes al poblamiento, propiedad de la tierra, fortalecimiento de la soberanía en un área de frontera y re direccionamiento de las actividades productivas (Bandieri, 2005, Blanco, 2008; Mendez, 2005; Navarro Floria, 2010). Paralelamente, se convirtió en un escenario de tensiones, conflictos y disputas de intereses, donde las lógicas super- 
puestas de los actores sociales, llevaron a privilegiar determinadas configuraciones territoriales y actividades económicas sobre las ya existentes. En este sentido, la capacidad que han tenido los actores hegemónicos -estatales y no estatales- de elaborar estrategias políticas para influenciar, dominar, controlar y gestionar el territorio, por sobre los modos de vida y derechos del pueblo mapuce, ha sido uno de los puntos álgidos de la historia regional.

Figura $\mathbf{N}^{\circ} 1$. Localización geográfica de la región de Los Lagos

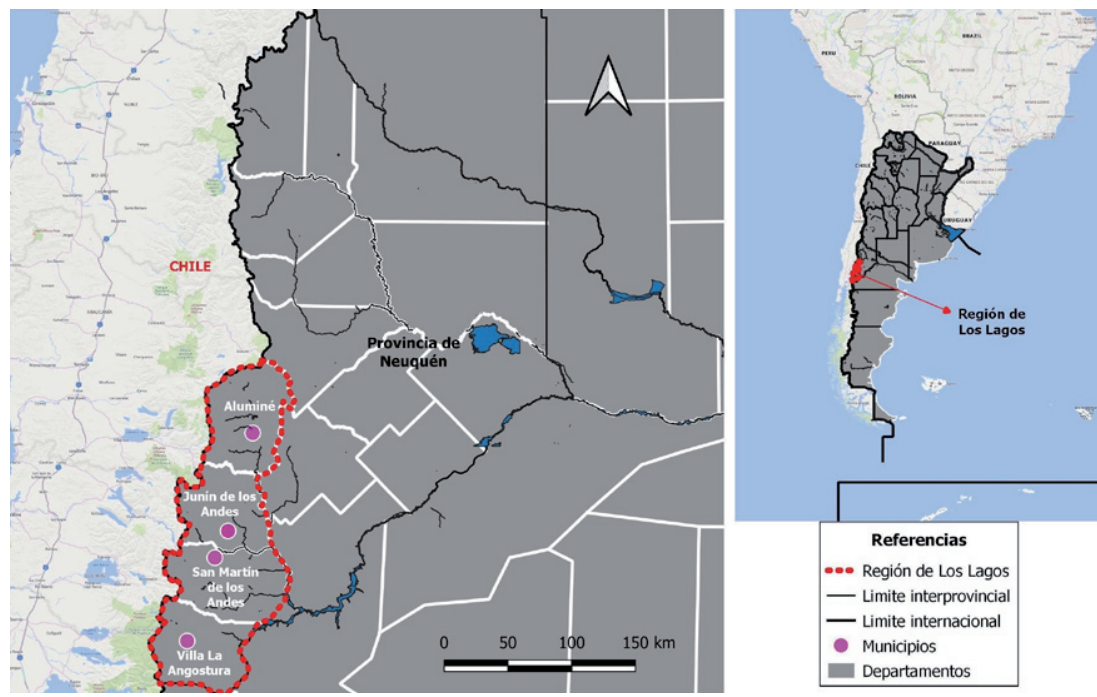

Fuente: Elaboración Cadiz Anabela en base a datos del Instituto Geográfico Nacional (IGN).

La región de Los Lagos, comienza a proyectarse como territorio turístico hacia los años 30', en concordancia con la creación de las áreas naturales protegidas en Argentina. A partir de ese momento, el turismo diseña y refuncionaliza el territorio, mediante la dotación de infraestructura física y de servicios, en tanto la naturaleza fue incorporada a la lógica de acumulación del capital y puesta al servicio de la demanda turística, para satisfacer las necesidades de ocio, esparcimiento y disfrute de los visitantes.

A medida que se consolida la región como destino turístico, hacia el interior se profundizan las desigualdades sociales heredadas de finales del siglo XIX. Basta indagar respecto a la política de distribución de la tierra, que fomenta el asentamiento del Estado o actores extra regionales, para darse cuenta que se trató de una política que favoreció a los grupos hegemónicos. Simultáneamente, se acentuó el despojo territorial de las 
comunidades mapuce, el no reconocimiento de los derechos de propiedad de la tierra y la exclusión de la toma de decisiones respecto al manejo y conservación de la naturaleza, quedando por fuera del desarrollo de las actividades turísticas. Es decir que, el Estado además de tener un rol protagónico en la reorganización de los territorios, desde finales del siglo XIX y la primera parte del siglo XX, ejecutó diversas estrategias para la exclusión del indígena como sujeto social y fomentó la construcción de una sociedad nacional monocultural. En tal sentido, la conformación de los nuevos estados estuvo dominada por la instalación de un Estado nación homogéneo y la conformación de fronteras físicas y políticas territoriales que sostuvieron la definición de los límites de una nación (Bengoa, 1994). El Estado nacional se conformó sobre la base de la exclusión del "otro/a", quienes sobrevivieron a dicho proceso tuvieron que asimilar el "ser argentino/a" para acceder a los derechos ciudadanos. Como puede verse, la desigualdad está presente desde los cimientos mismos de la conformación del Estado y la región estudiada no ha quedado exenta de este proceso.

El turismo llegó a la región para quedarse y fueron los actores hegemónicos (Estado y prestadores turísticos privados) quienes asumieron esa tarea. Hoy en día, la actividad tiene gran impacto en la región de Los Lagos dado que motoriza y es motorizada por otros sectores económicos (comercial, construcción, transporte) y contribuye con su demanda a la generación de empleos directos e indirectos. Sin embargo, la participación de los actores continúa siendo desigual y fragmentaria territorialmente, dado que se concentra básicamente en las ciudades cabeceras de los departamentos mencionados. Para ser más específico, San Martín de los Andes, cabecera del Departamento Lácar, es el principal centro turístico de la provincia. Con una población de 28.559 personas según el último censo nacional (INDEC, 2010), la mayor parte de la población económicamente activa se encuentra ocupada en el sector turístico. No obstante, la estacionalidad de la actividad, los eventuales fenómenos naturales que afectan su normal desarrollo, así como el alto grado de informalidad laboral y de alojamientos no autorizados, hacen del turismo un rubro difícil de controlar y más aún de protocolizar en tiempos como el actual.

Según los datos proporcionados por la Dirección Provincial de Estadística y Censos de la Provincia de Neuquén (Anuario Estadístico, 2019), para el año 2018, de un total de 23.998 plazas hoteleras en toda la provincia, el Departamento Lácar cuenta con 6.692 plazas, el Departamento Los Lagos 4.973; Huiliches 1.399 y Aluminé 1.917, es decir, el grueso de las camas de alojamiento está localizado principalmente en las ciudades de San Martín de los Andes y Villa La Angostura. Sin contabilizar los alojamientos extra hoteleros no registrados en las estadísticas oficiales, que según informantes claves del área de Turismo de San Martín de los 
Andes, para el año 2020 "duplica los números oficiales", sobre todo en temporada alta. Siguiendo con el análisis, según el informe de estimación de la PEA (población económicamente activa) realizado por el Ministerio de Turismo de Neuquén en marzo del año 2020, el 76\% de la PEA de San Martín de los Andes se ocupa en el sector privado, en el cual se destacan tres ramas de actividades económicas vinculadas al turismo (gastronomía, alojamiento, transporte) (Otero y Rodríguez, 2020).

Ahora bien, si revisamos las estadísticas oficiales para corroborar la existencia de experiencias turísticas de las comunidades mapuce, encontramos que, en los datos publicados por la Dirección Provincial de Estadística y Censos de la Provincia de Neuquén, hay un ítem que llamó la atención por varias cuestiones, el denominado "territorio rural comunitario mapuce". En primera instancia, se reconoce la insuficiencia de los datos, ya que solo cuenta con información de dos de los cuatro departamentos, de los cuales destaca Aluminé, que, de las 1.917 plazas hoteleras, 245 (12\%) forman parte del ítem turismo rural comunitario mapuce y en Los Lagos, de un total de 4.973, 124 (2,5\%), no registrándose datos en los departamentos Huiliches y Lácar, aunque existe la presencia de población mapuce y criolla en los mencionados departamentos. Lo que deja entrever la estadística es que hay una activa participación de las comunidades en el desarrollo de la actividad y aunque no esté contemplado oficialmente, existen emprendimientos turísticos administrados por las comunidades mapuce, en jurisdicción del Parque Nacional Lanín (Dpto. Huiliches), tales como el Camping Piedra Mala en el Lago Paimún, Camping Cañicul en el Lago Huechulafquen y Camping de la comunidad Curruhuinca en Quila Quina en el lago Lácar (Dpto. Lácar), solo por nombrar algunos ejemplos. Por otra parte, tampoco están contempladas las áreas de acampe, muchas de las cuales están gestionadas por las comunidades mapuce. Esto reafirma que existen experiencias turísticas en territorio mapuce administradas por las comunidades como forma de inserción socioeconómica para ubicarse de otro modo frente a la subalternidad.

En segunda instancia, el indicador construido por el organismo oficial genera algunas reflexiones. La denominación territorio rural abarca aquellas actividades económicas, particularmente turísticas recreativas que se realizan en el ámbito rural, contemplando una diversidad de manifestaciones que van desde el turismo en estancias a experiencias gastronómicas en un predio rural. En tanto, el término comunitario alude a la forma de organización del turismo. En este caso, la indicación mapuce da cuenta que el servicio turístico es desarrollado por las comunidades y se encuentra dentro del ámbito territorial indígena. En definitiva, surgen dudas en relación al criterio y la especificidad del indicador oficialmente publicado, dado que algunas características se refieren a lo meramente geográfico (el 
espacio rural), en otro caso a la forma de organización (comunitaria) y en otro al reconocimiento identitario y control territorial (mapuce).

No caben dudas que San Martín de los Andes y Villa La Angostura son los principales puntos de atracción al turista. En la temporada invernal, la posibilidad de realizar actividades de esquí y snowboard, convierten a los Cerros Chapelco y Bayo en los centros de esquí elegidos por el turismo. El caso del centro de esquí Cerro Chapelco ${ }^{5}$, concesionado desde el año 1987 a la empresa Nieves de Chapelco, ha sido foco de tensiones y conflictos socioterritoriales con parte de la localidad sanmartiniense. Importantes transformaciones territoriales generan un impacto social y un deterioro de los espacios de vida cotidiana, que no hacen más que profundizar la desigualdad existente en la región de Los Lagos. Otras localidades que conforman el entramado turístico en la región son Aluminé y Junín de los Andes. Ambas de menor población e infraestructura turística que las primeras mencionadas. Las mismas se destacan por un turismo estival vinculado al rafting en el caso de Aluminé y a la pesca y turismo religioso en Junín de los Andes. En este sentido, las características de la demanda también difieren en relación a San Martín de los Andes y La Angostura, dado que tampoco cuentan con centros de esquí para la temporada invernal. Sin embargo, en el verano, la demanda turística es alta a partir de su cercanía con lagos y ríos y por ubicarse como puerta de entrada a áreas de acampe del parque.

La configuración territorial creada en torno al turismo, sobre todo en los sitios antes mencionados, contribuyó a profundizar las desigualdades sociales, la fragmentación territorial y la exclusión de ciertos sectores de la población. Nos interesa la particularidad del pueblo mapuce y de las comunidades que habitan el territorio. Un pueblo que ha sufrido el despojo territorial por parte del Estado nacional y provincial, la persecución, estigmatización y criminalización por parte de las autoridades gubernamentales, el avasallamiento de los derechos territoriales por parte del capital privado y el revés de la justicia. Aun así, el Pueblo Mapuce ha podido reorganizarse y llevar a cabo la recuperación de los territorios y con ello las prácticas comunitarias, la lengua y los saberes ancestrales que dan identidad a la cultura mapuce (Delrio, 2017; Valverde, García y Berten, 2010; Trinchero, 2010). Las iniciativas y experiencias turísticas del pueblo mapuce en la provincia, tales como las desarrolladas por las comunidades Puel, Aigo, Ñorquinco, Cayulef, Currumil, Lefiman, Paicil Antriao, entre otras, han demostrado que es posible organizarse autónomamente para llevar a cabo el turismo en territorio mapuce, de forma colectiva, participativa y autogestionada atendiendo a los estándares de la economía social y solidaria. El fin no es el turismo masivo, ni consiste en realizar actividades recreativas de alto impacto, sino el resguardo del territorio, la protección 
del ambiente, la conservación del bosque nativo y las fuentes de agua, como forma de fortalecer la lucha, resistencia, construir la "re-existencia" (Porto-Goncalves, 2002) y establecer vínculos con diferentes actores.

En territorio neuquino, las comunidades originarias son una alternativa para el desarrollo del turismo. Por ello, recuperar dichas experiencias, ayudan a entender la lógica, expectativas, visiones y proyectos de vida que los impulsan como colectivo social. El escenario descrito, invita a repensar la construcción de los territorios turísticos en cuanto a la valorización de la naturaleza-cultura, con la idea de avanzar hacia una alternativa turística con igualdad de participación de todos los actores sociales involucrados. Una forma de acortar la brecha existente en el sector turístico, dar legitimidad a las voces y percepciones que tienen las comunidades mapuce respecto al uso, manejo del territorio y el desarrollo de actividades económicas.

\section{Una aproximación a las experiencias turísticas en la región de Los Lagos desde las acciones del pueblo mapuce}

"Los mapuce asumen plenamente que la proyección como pueblo originario requiere el resguardo de los territorios que se ocupan ancestralmente y el ejercicio de la autonomía como nación originaria" (Informe final de los resultados del relevamiento territorial histórico, social y cultural de la comunidad mapuce Lof Paichil Antriao, 2013, p.18). Es decir, el territorio es el espacio vivido y está cargado de historia, memoria, vivencias, saberes, sentires y pensares del ser mapuce. Además, constituye el ámbito político en el cual se toman autónomamente las decisiones colectivas respecto al uso y valorización de los recursos, así como los planes de vida. Es por ello que, la defensa de los derechos a través de la recuperación y reivindicación territorial, ocupan un lugar central en la condición de originarios del espacio que habitan. Territorios en los cuales se resisten ante el avance del capital privado y se convierten en protagonistas del proceso de re-existencia.

La colonialidad del ser, del saber, de la naturaleza y las formas de vida constituyeron la base estructural histórica, cultural y epistemológica sobre la que se asentó el proyecto moderno-colonial y civilizatorio de la Patagonia. Uno de los rasgos distintivos fue la dicotomía hombre-naturaleza y naturaleza-cultura. Ambas gestadas en torno a la separación ontológica sujeto-objeto, que coloca al hombre como "superior", capaz de dominar y controlar la naturaleza y convertirla en recursos naturales. De esta forma, la imposición de una racionalidad instrumental, economicista y utilitarista, transforma la naturaleza en un objeto de mercado al servicio de la dinámica impuesta por el modo de producción capitalista (Cadiz y Abar- 
zua, 2019). Contrariamente a la perspectiva anteriormente señalada, se encuentra la cosmovisión del pueblo mapuce, que en palabras de una referente dice "La naturaleza somos todos/as y es de todos/as, por eso tenemos que respetarla y cuidarla". En consonancia con lo planteado en los planes de vida (Kvme Felen), es necesario entender la articulación sociedad-naturaleza-cultura-identidad y aproximarse a la concepción de naturaleza como un referente de la simbolización y significación de las prácticas sociales, potencial de la riqueza material y soporte de la vida espiritual de los pueblos (Leff, 2004). Lo que equivale a decir que se desarrollan prácticas alternativas sustentadas en el conocimiento local y en la "estructuración de modelos culturales de la naturaleza" (Escobar, 2000, p.118). Resulta interesante del proceso mencionado que para las comunidades indígenas se convierte en una alternativa en la cual se combinan la "reconstrucción" colectiva de la cultura, el proceso de autonomía en la toma de decisiones, la visibilización de la identidad de un pueblo y una propuesta contrahegemónica al modelo de acumulación dominante.

En los últimos veinte años, surgieron experiencias de turismo en comunidades mapuce en la provincia. En la región de Los Lagos se resaltan servicios de alojamientos como campings organizados frente a los lagos Aluminé, Ruca Choroy y río Aluminé, gestión de parque de nieve en el Cerro Batea Mahuida (Villa Pehuenia), prestación de servicios gastronómicos, camping, servicio de cabalgata o venta de artesanías en la Cuenca Huechulafquen $^{6}$ (Parque Nacional Lanín), área de acampe, servicio gastronómico y actividades náuticas en el lago Correntoso (Villa La Angostura), alquiler de equipamiento en la base del Cerro Chapelco y área de uso diurno en lago Lácar (San Martín de los Andes). Estos son algunos ejemplos que reconocen el desenvolvimiento del turismo con un acceso y uso de bienes naturales en territorios de comunidades mapuce. A continuación, se abordan experiencias turísticas desarrolladas por las comunidades mapuce en el centro, norte y sur de la región de los Lagos, recabadas a partir del trabajo de campo.

\section{Experiencias mapuce de turismo en el centro de la región de Los Lagos}

En la zona centro de la región de Los Lagos, específicamente en el centro y norte del Parque y la Reserva Nacional Lanín (PNL) habitan en la actualidad, aproximadamente entre 2.500 y 3.000 pobladores mapuce, que pertenecen a siete comunidades (Aigo, Lefimán, Ñorquinko, Cañicul, Raquithue, Curruhuinca y Cayún) ocupando unas 24.000 hectáreas del área protegida (Rodríguez, 2017). Esta población se dedica a la cría de ganado (ovino, vacuno, caprino y equino) a través de un modelo ganadero trashumante y estacional, a la forestación y recientemente una in- 
cipiente prestación de servicios turísticos. En el año 2012, a partir de un relevamiento realizado por un equipo de la Facultad de Turismo de la Universidad Nacional del Comahue ${ }^{7}$, se identificaron treinta y dos emprendimientos turísticos mapuce ubicados en la zona de incumbencia, veinticuatro en la cuenca Huechulafquen, siete en la costa del río Malleo y dos emprendimientos en Tromen. Los emprendimientos presentan grandes diferencias entre sí, en relación al tipo y cantidad de servicios que prestan, la cantidad de personas que trabajan, la forma de organización, el desarrollo de la infraestructura y las tecnologías aplicadas, poniendo de manifiesto la complejidad al momento de trabajarlas.

\section{Experiencias mapuce de turismo en el norte de la región de los Lagos}

Desde el año 2000, en las cuencas de Ruca Choroy -asentamiento de las comunidades Aigo y Ninhigual-, la cuenca Quillen -las comunidades Currumil y Lefiman-, y en la comunidad Norquinco, del Departamento Aluminé, se presentan experiencias en la temporada estival a partir de campings libres y organizados. Estas familias combinan la actividad con emprendimientos como la venta de artesanías, alimentos -carne, pan, alfajores de piñón, etc- y alquiler de bicicletas y cabalgatas. Otra experiencia es la administración y gestión del Parque de Nieve Batea Mahuida en la comunidad Puel (Villa Pehuenia), que desde inicios del año 2003 sostiene un proyecto turístico de gestión autónoma en la temporada invernal. Se trata de experiencias laborales de organización y participación del pueblo mapuce en el circuito productivo local-regional. Esto se da en un contexto de reconversión de las estructuras agrarias regionales, los procesos de privatización de la tierra, acceso restringido a la costa de los lagos, despojo territorial y relocalización de población, migración hacia centros urbanos y zonas periurbanas y redefinición de las prácticas productivas de las unidades domésticas.

En el Departamento Aluminé, se registra que el acampe espontáneo de turistas generó el surgimiento de campings agrestes y organizados gestionados por familias de la comunidad mapuce Catalán ubicadas a la vera del río Aluminé. Por su parte, un grupo de mujeres de dicha comunidad creó un proyecto denominado Quimey Mapu que agrupa a artesanas, con el fin de ofrecer sus productos al turista. La conflictividad está latente en este territorio, dado que algunas agencias de turismo de Villa Pehuenia venden servicios de pesca, rafting sobre el río, construyen muelles precarios e ingresan al territorio sin previo aviso y consentimiento de la comunidad Catalán. La cercanía con el centro turístico de Villa Pehuenia genera una presión de prestadores turísticos privados por promover áreas de alto valor paisajístico en territorios de esta comunidad e influye en las familias colin- 
dantes al río Aluminé. Esta actividad ha generado cambios hacia el interior de la comunidad, en una relación diferencial entre: jóvenes y mayores, hombres y mujeres, mapuce y no mapuce, quienes trabajan en las actividades y quienes quedan al margen. Del trabajo de campo en las cuencas Quillen, Ruca Choroi y Ñorquinco en las temporadas estivales de los años 2017, 2018 y 2019 se identificaron 15 emprendimientos asociados al turismo en temporada estival. En los emprendimientos se emplean alrededor de 65 personas con modalidades de administración de los emprendimientos diversas según cada cuenca (familiar, comunitario, individual) y a la vez se da la particularidad de la administración del camping en la cuenca Quillen bajo la órbita del Consejo Zonal Pehuenche ${ }^{8}$.

\section{Experiencias mapuce de turismo en el sur de la región de Los Lagos}

Hacia el sur de la región estudiada, el trabajo de campo realizado en 2018-2019 en la comunidad Paicil Antriao (lindante a la localidad de Villa La Angostura), da cuenta que la población se encuentra dispersa territorialmente en 125 hectáreas $^{9}$, repartidas entre sector de Puerto Elma (a orillas del Lago Nahuel Huapi), la costa del lago Correntoso (camping Correntoso), el sector del Belvedere y el sector Picunko. Dicho territorio alberga aproximadamente un total de 127 familias, de las cuales 60 viven en territorio comunitario y el resto se encuentra repartida en los barrios más pobres de la ciudad (Barrios Mallín y Margaritas) (Informe final de los resultados del Relevamiento Territorial, histórico, social y cultural de la comunidad mapuce Lof Paicil Antriao, 2013). En la comunidad, puede observarse el "deterioro sustantivo de su hábitat en términos fundamentalmente de hacinamiento, calidad de la vivienda y del entorno por ausencia de infraestructuras de saneamiento" (Secretaria de Turismo de Villa La Angostura, 2016, p13), dificultades para el aprovisionamiento de energía eléctrica, agua potable y gas natural. Además, la asignación de la propiedad privada de la tierra y el rol del Estado en la promoción de políticas de inversión destinadas a fines turísticos, inmobiliarios y especulativos, desestructuró el modo de vida tradicional, comunitario y de libre circulación por los territorios de la comunidad y contribuyó a profundizar la brecha de la desigualdad. De todas formas, más allá de los problemas emergentes que enfrenta la comunidad ante la falta de reconocimiento de los derechos territoriales, la conflictiva relación con el municipio, el gobierno provincial y propietarios privados, uno de sus propósitos es convertirse en protagonistas del circuito turístico local. Allí surge la iniciativa de impulsar autónomamente una experiencia turística en el marco del Kvme Felen (Plan de Vida), sustentado en el trabajo comunitario y en la visibilización como pueblo originario. 


\section{Construyendo puentes hacia un turismo con mayor participación mapuce}

Se sostiene que el turismo surge como una actividad alternativa y estacional, que involucra a diversos actores sociales y dinamiza la economía en centros urbanos como San Martín de los Andes y Junín de los Andes, Villa La Angostura, Aluminé y Villa Pehuenia. Desde el año 2000 y en consonancia con el establecimiento de una mesa mixta de negociación que involucró a la Administración de Parques Nacionales, la Intendencia del Parque Nacional Lanín, el Instituto de Asuntos Indígenas, la Confederación Mapuce Neuquina y representantes de cada una de las comunidades de la región de Los Lagos, se adopta la práctica política denominada comanejo. Este hecho significativo permitió trabajar de manera conjunta la gestión del territorio y la resolución de conflictos y abrió el juego para que las comunidades se inserten en la actividad turística de diversas formas. Algunas de ellas sostienen emprendimientos ya consolidados en el territorio y otras, proyectos emergentes. Se afirma que, la inserción del turismo en el pueblo mapuce se produce de manera diversa a partir del acceso a los recursos, la dinámica social y económica de los grupos étnicos y su capacidad de agenciamiento en la construcción/ reproducción de territorios turísticos.

\section{Impactos socioeconómicos de la pandemia en los territorios turísticos de la región de Los Lagos}

La coyuntura actual vino a profundizar las desigualdades sociales existentes en los territorios, lo cual es traducible en el incremento de la pobreza y desigualdad en diferentes regiones y sectores sociales. En tal sentido, el interrogante que surge es: en un contexto de desigualdad manifiesta, como el actual, ¿qué papel juega el turismo en el territorio? La retracción económica ocasionada por la pandemia, impactó en todas las actividades y el turismo no fue ajeno a esa realidad. En un momento en el cual las políticas públicas a nivel provincial se orientan al fomento del turismo de proximidad o por microrregiones como propuesta para dinamizar la actividad, cabe el siguiente interrogante: ¿cómo se incorpora el turismo en la proyección de las políticas estatales para los territorios mapuce?

Se reconocen diversas miradas respecto a los impactos socioeconómicos de la pandemia en el turismo en la región de Los Lagos. En este contexto, ser un destino de relevancia como San Martín de los Andes o Villa La Angostura y además uno de los sitios elegidos por los turistas en la temporada estival, implica mayor riesgo desde el punto de vista epidemiológico. Además, hay que tener presente que ambas localidades actúan 
como un corredor de conectividad en un sistema de relaciones y flujos de movilidad.

Según consultas realizadas a informantes del organismo Parque Nacional Lanín, la institución tuvo un rol facilitador en la tramitación de asignaciones a familias que habitan en el ejido del parque, por ejemplo en la gestión del ingreso familiar de emergencia (IFE) ${ }^{10}$. Contrariamente, los servicios comunitarios o prestaciones informales no fueron contemplados y esto dificulta la posibilidad de acceder a los créditos dados por el Ministerio de Turismo Provincial. Algunas de las familias mapuce identifican la falta de herramientas tecnológicas y de infraestructura suficientes para la prestación de servicios turísticos en este contexto. Sumado a ello, la dificultad de acceso a servicios básicos como electricidad o internet en este territorio, repercute en una desventaja al momento de solicitar subsidios al Estado para los sectores más necesitados. Con respecto a la organización de las actividades para la pretemporada, tampoco se realizó por falta de manejo de herramientas digitales, falta de internet, electricidad, restricciones de circulación y solamente se realizaron reuniones presenciales, cuando fue posible, con algunos prestadores turísticos y referentes de las comunidades. Se trabajó coordinadamente en la reactivación de la zona del volcán Lanín, sobretodo en la regulación de las actividades de ascenso y en la manera más óptima de reactivar esos servicios. Se manifestó una gran preocupación en garantizar el cumplimiento de los protocolos en la temporada de verano y al mismo tiempo, generar condiciones para controlar y manejar en el territorio. Algunas de las ventajas de la virtualidad fueron las capacitaciones a los guías del parque, que según reglamento debe realizarse cada tres años (Otero y Rodríguez; 2020).

Los y las entrevistados/as ${ }^{11}$ reconocen un tratamiento diferencial en el acceso a los créditos ofrecidos desde el Estado. Muchos actores de las comunidades quedaron afuera de la oportunidad de acceder a la ayuda por no ser monotributistas y por dificultades de acceso a la tecnología. Por otro lado, manifiestan que los créditos ofrecidos desde el Ministerio de Turismo tuvieron una escasa duración de la inscripción, así como de difusión, lo cual dio por resultado que varios prestadores comunitarios no llegarán a tiempo para solicitarlos. Sumado a ello un obstáculo en el área rural representó que dicha inscripción fue exclusivamente a través de plataformas digitales. En relación a las ayudas económicas desplegadas por el Estado y su concreción con los actores del territorio, se puede mencionar que, de los diversos sectores prestadores de servicios en el territorio del PNL, las agencias de viajes, tramitaron la ayuda para sus empleados y pudieron acceder a esos créditos, pero no tuvo un alcance en la asistencia en el ámbito rural. 
Se acuerda con lo expresado por Otero y Rodríguez (2020), que la trama territorial del parque puede inferir que los impactos de la pandemia en el sector turístico han acentuado las desigualdades que ya existían en un contexto pre pandémico. Lo dicho actúa directamente en las dificultades por la disminución y recuperación del nivel de ingresos familiares. Algunas comunidades mapuce localizadas en la jurisdicción del parque mencionado decidieron cerrar su territorio al uso turístico durante este periodo, no así otras que abrieron sus puertas la primera semana de diciembre y fueron los lugares mayormente visitados en la temporada alta, por la posibilidad de acampar y realizar actividades al aire libre, disminuyendo los riesgos de contagio.

Hacia fines del año 2020, el escenario de apertura progresiva de la temporada estival, atendiendo a las particularidades de cada provincia, así como el establecimiento de "medidas y protocolos para el sector turístico en el contexto de la emergencia sanitaria" Ilevadas a cabo por el Ministerio de Turismo y Deportes de la Nación ${ }^{12}$ y el plan para la reanudación de los vuelos nacionales e internacionales, tensionaron las miradas de la sociedad en torno a la vuelta del turismo en la nueva normalidad. Dado que el flujo de turistas en la región de Los Lagos implica el ingreso de personas desde otros puntos del país, genera mayor movilidad entre los centros turísticos, así como concentración de personas, el relajamiento de las medidas sanitarias, todos elementos que favorecen la propagación del virus. A modo de compensación, el Ministerio de Turismo y Deportes de Nación dotó a los distintos centros turísticos del país de hospitales modulares para atender pacientes leves, ampliando la oferta de camas disponibles, incorporando personal de salud, reforzando las unidades de terapia intensiva, entre otras medidas de acción inmediata ${ }^{13}$.

En la región de Los Lagos, según el registro de campo, se observa: 1- un incremento del flujo de turistas que visitan la región; 2- distinción entre turistas que eligen acampar en sitios habilitados y aquellos que eligen hacerlo en centros urbanos; 3- A nivel nacional destaca la presencia de turistas provenientes de las provincias de Córdoba, Buenos Aires y Mendoza; 3- A nivel regional destaca la presencia de turistas provenientes del Alto Valle de Río Negro y Neuquén; 4- Los turistas mayormente cumplen los requisitos de ingreso a la localidad (reserva previa, declaración jurada, permiso de circulación, seguro de viaje); 5- San Martín de los Andes y Villa La Angostura figuran como los destinos más elegidos para vacacionar; 6- La información suministrada por los medios de comunicación regionales, expresa la demanda turística centrada en San Martín de los Andes y Villa La Angostura y una escasa atención al resto de la región. De todas formas, habrá que esperar la finalización de la temporada para hacer un balance del turismo y proyectar los pasos a seguir ante un escenario todavía incierto. 


\section{Reflexiones finales}

A lo largo del trabajo se ha demostrado que la configuración territorial en la región de Los Lagos creada en torno al turismo contribuye a profundizar las desigualdades socioeconómicas, la fragmentación territorial y la exclusión de ciertos sectores de la población, entre ellos el pueblo mapuce. Una diferencia social, económica y territorial que se torna histórica y estructural, en la medida que el Estado a través de dispositivos políticos-normativos consolida un modelo turístico selectivo; a la vez que fomenta la participación del capital privado y estatal en el desarrollo de la actividad por un lado y restringe o limita la inserción de los actores subalternos por otro.

Sin embargo, este panorama plagado de incertidumbre y planificación a corto plazo lleva a retomar los interrogantes planteados al inicio del trabajo y reflexionar que en la región de Los Lagos la especificidad y/o diversidad indica que la actividad es desarrollada mayormente por el Estado y prestadores turísticos, entre los cuales se encuentran las comunidades mapuce. Las experiencias turísticas que llevan adelante las comunidades bajo la denominación turismo con identidad o turismo en territorio mapuce, demostraron que esta actividad es sostenida hace varias décadas por el pueblo mapuce. Asimismo, se concibe a la misma como una estrategia para proyectarse en el territorio, complementar con la economía doméstica familiar, avanzar hacia la articulación social y reafirmar los derechos ancestrales. Las experiencias tienen como denominador común alternativa de inserción laboral en el territorio ante la expropiación del capital y despojo territorial.

Para un balance global post-pandemia habrá que esperar a que finalice la misma y se puedan procesar los datos de las temporadas invernales y estivales acontecidas. Aunque sí se pueden esbozar algunas reflexiones en torno a lo transitado desde el inicio. A nivel general, las estadísticas mundiales y regionales afirman que la población pobre agudizó su condición económica con la pandemia.

Claramente, el escenario de emergencia producto de la pandemia pone en evidencia que las políticas públicas nacionales y provinciales no contemplaron, principalmente en las ayudas económicas, al grupo minoritario representado por el pueblo mapuce. $\mathrm{Y}$ estos actores, debieron recurrir a reforzar las redes de vinculación con instituciones como Parques Nacionales u organizaciones no gubernamentales para generar mecanismos que le permitan una articulación en un momento de crisis como el actual. Es decir, el COVID-19 advierte que la desigualdad se acentuó y estos sectores continúan siendo excluidos por el Estado. Por otro lado, las dificultades para crear o consensuar protocolos sanitarios en una actividad que se manifiesta en un gran porcentaje al aire libre, es otra de los elementos que 
se manifiestan en la articulación entre sectores. No obstante, se puede observar cierto fortalecimiento interinstitucional de redes, y en el caso del pueblo mapuce, la posibilidad ante la pandemia, de volver a participar en redes, mesas y comités que accionaron y ejecutaron decisiones en el territorio. En este sentido, la voz, la participación y la toma de decisiones por parte de actores del pueblo mapuce se reconoce como una oportunidad en el contexto actual.

Finalmente, "la nueva normalidad", tal vez pueda tomarse como una oportunidad para incentivar, impulsar o potenciar estrategias vinculadas al turismo no masivo, que revalorice el cuidado del ambiente, que reconozca los mapuce como pueblo preexistente localizados en territorios dotados de atractivos turísticos y por último, otorgue a las comunidades mapuce un papel central en la toma de decisiones y la ejecución de acciones. Sería sumamente válido que, en la proyección de escenarios futuros para el turismo, puedan incorporarse cosmovisiones no occidentales para abordar esta actividad y como expresa Zusman, et. al. (2020, p.246) "pueden contribuir no solo al de-aprendizaje de los lineamientos del modelo de turismo actual, sino que también pueden colaborar en la incorporación de nuevos enfoques y valores orientados al cambio".

\section{Referencias bibliográficas}

Albert i Mas, A. (2001). “iRegiones singulares y regiones sin lugares? Reconsiderando el estudio de lo regional y lo local en el contexto de la Geografía postmoderna", en Boletín de la A.G.E. No 32, Madrid. pp. 3552. Recuperado de: https://dialnet.unirioja. es/servlet/articulo?codigo $=1122497$

Bandieri, S. (2005) Historia de la Patagonia. Buenos Aires. Editorial Sudamericana.

Bengoa, J. (1994) La historia del pueblo Mapuche. Santiago de Chile. Ediciones SUR, julio, $3^{a}$ edición.

Blanco, G. (2008) "La disputa por la tierra en la Patagonia norte. Ganadería, turismo y apropiación de recursos naturales en Neuquén a lo largo del siglo XX". Revista digital de la Escuela de Historia. Universidad Nacional de Rosario. AÑO 1 No 2. Rosario, agosto-diciembre 2008, ISSN 1851-992X.

Cadiz, A. y Abarzua, F. (2019). Diálogos entre la Geografía y la Ecología Política. Aportes para repensar los conflictos territoriales en la Norpatagonia. VII Congreso Nacional de Geografía de Universidades Públicas y XXI Jornadas de Geografía de la UNLP. Recuperado de http://jornadasgeograa.fahce.unlp.edu.ar

Contino, G. (19/8/2020) ¿Cómo es el turismo por Microrregiones que permitió la llegada de turistas a Villa La Angostura? Disponible en https://www.anbariloche.com. ar/noticias/2020/08/19/76395-como-es-elturismo-por-microrregiones-que-permitio-lallegada-de-turistas-a-villa-la-angostura

Gobierno de la Provincia de Neuquén http:// neuquentur.gob.ar/

De Jong, G. (2009). Geografía, Método Regional y Planificación. Ed. Catálogos. Bs As.

Delrio, W. (2017). ¿A qué se Ilama Conquista del Desierto? Ciencia Hoy, Vol. 26, No 156. Pp. 45-51.

El Intransigente 27/2/2021 "Matías Lammens resaltó que la temporada de verano 
fue "muy exitosa» Recuperado de https:// elintransigente.com/2021/02/matias-lammens-resalto-que-la-temporada-de-veranofue-muy-exitosa/

Dirección Provincial de Estadística y Censo (2019) Anuario Estadístico. Provincia de Neuquén.

Escobar, A. (2000). El lugar de la naturaleza y la naturaleza del lugar: iglobalización o postdesarrollo? En la colonialidad del saber: eurocentrismo y ciencias sociales. Perspectivas Latinoamericanas. Bs. As. CLACSO.

Gordillo, G. y Hirsch, S. (2010) Movilizaciones indígenas e identidades en disputa en la Argentina". La Crujía. Buenos Aires.

INDEC (2010) Datos generales de población y vivienda. Buenos Aires.

Informe final de los resultados del Relevamiento Territorial, histórico, social y cultural de la comunidad mapuce Lof Paicil Antriao, (2013). ODHPI. Huellas y senderos. Neuquén (Puel Mapu. Recuperado de http://biblioteca.clacso.edu.ar/clacso/ gt/20100817091913/saberes.pdf

Lof Paicil Antriao. (s/f)El Buen vivir desde territorio mapuce. La experiencia de pul of Kintupuray, Kinxikew y Paicil Antriaw, s/f.

Leff, E. (2004). Racionalidad ambiental: la reapropiación social de la naturaleza. Siglo XXI editores. Argentina. Pp. 352- 395

March, Hug (2013). Neoliberalismo y medio ambiente: una aproximación desde la geografía crítica. Documents d'Anàlisi Geog ràfica, vol. 59/1 137-153. Recuperado de: https://www.researchgate.net/ publication/258416385_Neoliberalismo_y_ medio_ambiente_una_aproximacion_desde_la_geografia_critica

Mendez, L. (2005). Circuitos económicos en el gran lago. La Región del Nahuel Huapi entre 1880 y 1930. En Bandieri, S.; Blanco, G. y Varela, G. (Directoras) Hecho en Patagonia. La historia en perspectiva regional. Educo. Neuquén. Argentina.

Navarro Floria, P. (2010). La conquista de la patagonia y el reparto de tierras en Historias de las Familias Mapuche Lof Paichil Antriao y Lof Quintriqueo. Mapuche de la margen Norte del Lago Nahuel Huapi. Archivos del Sur. Biblioteca Osvaldo Bayer. Córdoba.

Nataine, D.; Fernàndez Moujan, M.; Rodríguez, M.D.; Karlau; A.; Cabrera, M.; Scarabotti, C. (2012) Proyecto de Desarrollo Rural del Comitè Huiliches. Informe técnico. FATU-CRUB-UNCO. Junín de los Andes, Neuquén.

Noticias de Neuquén 16/02/2021 Ocupación plena en la mayoría de los destinos turísticos neuquinos. Recuperado de https:// www. noticiasdeneuquen.com.ar/ocupacionplena-en-la-mayoria-de-los-destinos-turisticos-neuquinos/

Otero, A. y Rodriguez, M. D. (2020) El turismo y las movilidades contemporáneas en un tiempo de transición: en los campos disciplinares y en las configuraciones materiales. En Turismo y Recreación Post COVID-19. Perspectivas, reflexiones y propuestas para una nueva realidad del campo disciplinar. (En prensa). Facultad de Turismo, UNC. Neuquén.

Secretaría de Turismo de Villa La Angostura (2016). Plan de Desarrollo y promoción turística de Villa La Angostura. Gobierno de la provincia del Neuquén.

Porto-Gonçalves, C. (2002) Da geografia ás geo-grafías: um mundo em busca de novas territorialidades. En: Ceceña, A. E. y Sader, E. (Coord.) La guerra infinita. Hegemonía y terror mundial. Buenos Aires, CLACSO.

Resolución Provincial 56/20. Provincia de Neuquén.

Rodríguez, M. D. (2017) Tesis doctoral. Territorio en disputa: turismo y forestación en las comunidades mapuche Puel y Catalán en la provincia de Neuquén. Universidad Nacional de Quilmes. Buenos Aires.

Rodríguez, M.y Merlos, M. (2020). Estrategias territoriales en la norpatagonia andina en un contexto de desigualdades. Boletín Geográfico, 42 (2), 35-56. Recuperado de: http://revele.uncoma.edu.ar/htdoc/revele/index.php/geografia/article/view/3034 
Telam 19/02/2021. La temporada de verano contabiliza 11 millones de turistas en el país. Recuperado de https://www.telam. com.ar/notas/202102/545111-la-temporada-de-verano-contabiliza-11-millo

Trinchero, H. (2010). Los pueblos originarios en Argentina. Representaciones para una caracterización problemática. Cultura y representaciones sociales. México D.F.; p. 111-139.

Valenzuela C. (2012). Enfoques y conceptualizaciones de la región en el contexto multiparadigmático de la Geografía contemporánea. Recuperado de observatoriogeografiaamericalatina.org.mx pp.1-10 pdf

Valverde, S.; García, A. y Berten, L. (2010). Mitos, prejuicios y realidades sobre los mapuches en el Departamento los Lagos, Provincia de Neuquén en Historias de las Familias Mapuche Lof Paichil Antriao Lof Quintriqueo. Mapuche de la margen Norte del Lago Nahuel Huapi. Archivos del Sur. Biblioteca Osvaldo Bayer. Córdoba.

Zusman, P.; Bietti, G.; Landini, G. (2020) Las múltiples implicancias espaciales de la difusión del COVID-19. Un estado de la cuestión. Notas de Coyuntura. Punto Sur 3 (Julio-Diciembre, 2020) Pp 234-262. doi:10.34096/ps.n3.9707

\section{Notas}

1 María Daniela Rodríguez, Lic. en Turismo, Dra. En Ciencias Sociales. Investigadora del IPEHCS- CONICET-UNCo. Docente de la FATU, UNCo. Integrante del Proyecto de investigación NEU 5 "Desigualdades e impactos socio-económicos del COVID 19 en la provincia de Neuquén" del IPEHCS-CONICETUNCo y financiado por el Programa de Articulación y Fortalecimiento Federal de las capacidades en Ciencias Tecnología COVID 19 del Ministerio de Ciencia, Tecnología e Innovación de la República Argentina. Integrante del Proyecto Unidad Ejecutora "La (re)producción de las desigualdades en la Patagonia Nor- te. Un abordaje multidimensional" del IPEHCS-CONICET-UNCO.

Anabela Ivana Cádiz. Profesora en Geografía. Doctoranda en Geografía (UNS). Becaria doctoral del IPEHCS-CONICETUNCo. Docente e investigadora Dpto. de Geografía. FaHu. UNCo. Integrante del Proyecto Unidad Ejecutora "La (re)producción de las desigualdades en la Patagonia Norte. Un abordaje multidimensional" del IPEHCS-CONICET-UNCo.

2 Se aclara que el término corredor, es una denominación turística que sirve para identificar un área geográfica, cuyo trayecto es de $220 \mathrm{~km}$ desde la ciudad de San Martín de los Andes a la localidad de Villa La Angostura, provincia de Neuquén. En su recorrido se observan siete lagos: Machónico, Falkner, Villarino, Escondido, Correntoso, Espejo y Nahuel Huapi, de ahí que también sea conocida como ruta de los siete lagos.

3 Proyecto "Desigualdades e impactos socio-económicos del COVID19 en la provincia de Neuquén" dirigido por la Dra. Verónica Trpin. IPEHCS/CONICET/ UNCO, del Ministerio de Ciencia y Tecnología. Año 2020.

4 En la investigación se incorporan los aportes de la Geografía Crítica, la Historia Regional, la Ecología Política, la Antropología.

5 “En el año 1973 el Estado concesionó el centro de esquí Chapelco a la empresa Sol Jet -propietaria de la línea aérea Austral- que promociona y posiciona a San Martín de los Andes como destino para un nuevo público, los esquiadores. Desde esta época, el centro de esquí estuvo atado al negocio inmobiliario a través del loteo "Aldea del Sol". (Rodríguez, Merlos; 2021, p. 45).

6 La Cuenca Huechulafquen se ubica en el Parque Nacional Lanín en el cual habitan poblaciones de las comunidades Lafquenche y Raquitue. El centro urbano más cercano es Junín de los Andes, en la zona sur de la provincia.

7 Programa de Desarrollo Rural Comité Huiliches. Fatu- Crub/UNCo y Consejo 
Zonal Huiliches. Equipo técnico: Mg. Juan Daniel Nataine, Lic. María Fernández Moujan, Dra. María Daniela Rodríguez, Lic. Alejandro Karlau, Lic. María Cabrera, Lic. Celeste Scarabotti.

8 Organización política territorial del pueblo mapuce. El Consejo Zonal Pehuenche está conformado por las autoridades de las comunidades Puel, Catalán, Lefiman, Currumil, Aigo, Norquinco y Ninhigual.

9 En 1902 concedió, mediante decreto 625 hectáreas del lote $N^{\circ} 9$ de la CoIonia Agrícola Pastoril Nahuel Huapi a los jefes de familia José María Paichil y Juan Ignacio Antriao. Se trata del área ocupada actualmente por el ejido urbano de Villa La Angostura. Posteriormente el avance de la propiedad privada, el engaño y despojo territorial contribuye a la reducción de la superficie originalmente otorgada a la comunidad, que hoy ocupa 125 hectáreas.

10 "El Ingreso Familiar de Emergencia (IFE) es una medida excepcional implementada por el Gobierno Nacional, que busca proteger a las familias argentinas ante la pérdida o disminución de sus ingresos por la situación de emergencia sanitaria generada por el COVID-19" (https:// www.anses.gob.ar/informacion/ingresofamiliar-de-emergencia)

11 Trabajadores/as de Parques Nacional Lanín y referentes de las comunidades mapuce: Paicil Antriao, Catalan, Currumil.

12 Para mayor información revisar "Medidas en turismo frente al COVID-19", Ministerio de Turismo y Deportes de la Nación disponible en https://www.argentina.gob.ar/turismoydeportes/medidas-en-turismo-frente-al-covid-19

13 En San Martín de los Andes implicó una oferta de diez nuevas camas hospitalarias. 